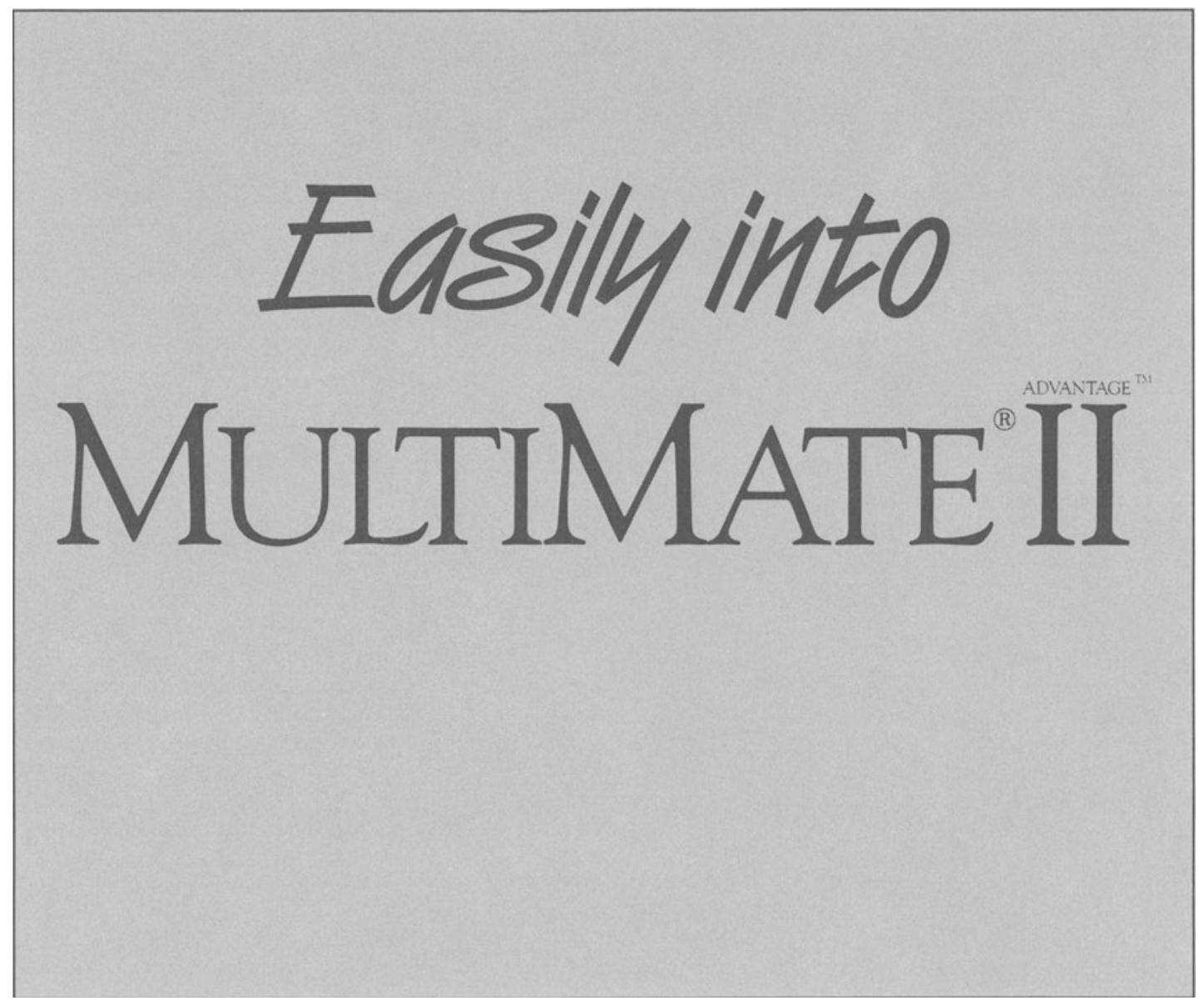

\title{
Joanna Gosling
}

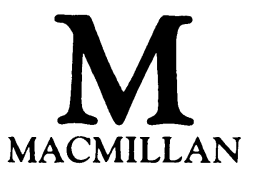


All rights reserved. No reproduction, copy or transmission of this publication may be made without written permission.

No paragraph of this publication may be reproduced, copied or transmitted save with written permission or in accordance with the provisions of the Copyright Act 1956 (as amended), or under the terms of any licence permitting limited copying issued by the Copyright Licensing Agency, 33-4 Alfred Place, London WC1E 7DP.

Any person who does any unauthorised act in relation to this publication may be liable to criminal prosecution and civil claims for damages.

First published 1989

Published by

MACMILLAN EDUCATION LTD

Houndmills, Basingstoke, Hampshire RG21 2XS

and London

Companies and representatives

throughout the world

British Library Cataloguing in Publication Data

Gosling, Joanna

Easily into MultiMate Advantage II.

(Macmillan modern office).

1. Word processing. Applications of

microcomputer systems. Software packages.

MultiMate Advantage II

I. Title

$652.5^{\prime} 02855369$

ISBN 978-1-349-10487-1 ISBN 978-1-349-10485-7 (eBook)

DOI 10.1007/978-1-349-10485-7 


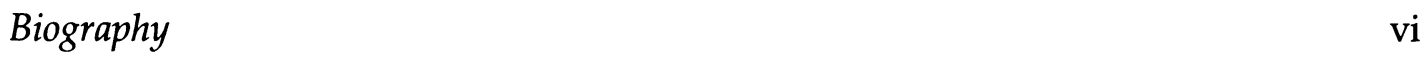

Acknowledgements vii

What this book aims to do 1

Before you start $\quad 2$

Lesson One: Creating and Printing a Simple Letter 4

$\begin{array}{ll}\text { Starting up } & 5\end{array}$

Making selections from menus $\quad 8$

$\begin{array}{lr}\text { Creating a document } & 8\end{array}$

Naming documents 9

Workscreen $\quad 11$

Status line $\quad 12$

Format line $\quad 12$

$\begin{array}{ll}\text { Keyboard } & 13\end{array}$

Keying-in text $\quad 14$

$\begin{array}{ll}\text { Moving the cursor } & 15\end{array}$

Inserting text $\quad 16$

Deleting text $\quad 17$

$\begin{array}{ll}\text { Getting help } & 19\end{array}$

$\begin{array}{ll}\text { Printing your document from the screen } & 21\end{array}$

$\begin{array}{ll}\text { Saving your document } & 21\end{array}$

Leaving MultiMate Advantage II $\quad 21$

Notes on defaults $\quad 22$

Lesson Two: Recalling and Editing the Simple Letter 24

Editing a document $\quad 24$

$\begin{array}{ll}\text { Altering words } & 26\end{array}$

$\begin{array}{ll}\text { Inserting blank lines } & 26\end{array}$

$\begin{array}{ll}\text { Changing margins using the menu } & 27\end{array}$

Changing the margins using F9 29

Saving your document using the menu $\quad 30$

Printing from the Opening Menu $\quad 32$

Document print options $\quad 33$

$\begin{array}{ll}\text { Cancel print } & 38\end{array}$

$\begin{array}{ll}\text { Pause print } & 39\end{array}$

Lesson Three: Creating, Printing and Editing an Invoice 40

Clearing tabs $\quad 42$

Setting tabs $\quad 43$

Centring lines $\quad 44$

Using tabs and decimal tabs $\quad 45$

$\begin{array}{ll}\text { Making alterations to a table } & 48\end{array}$

$\begin{array}{ll}\text { Hotprinting } & 49\end{array}$

Underlining existing text $\quad 50$

Saving and printing from the menu $\quad 51$ 
Lesson Four: Creating and Printing a Table of Figures 52

Clearing tabs $\quad 53$

Setting tabs $\quad 54$

Centring and underlining text as you type it $\quad 55$

Using tabs $\quad 57$

Saving and printing from the menu $\quad 58$

Lesson Five: Using Indent, Line Spacing and Justification $\quad 60$

$\begin{array}{ll}\text { Clearing tabs } & 62 \\ \end{array}$

Setting tabs $\quad 62$

Setting the right margin $\quad 62$

Underlining text as you type it $\quad 63$

$\begin{array}{ll}\text { Indenting text to first tab } & 64\end{array}$

Indenting text to second tab $\quad 64$

$\begin{array}{ll}\text { Hotprinting } & 65\end{array}$

Removing one level of indentation $\quad 66$

$\begin{array}{ll}\text { Hotprinting } & 67\end{array}$

$\begin{array}{ll}\text { Closing text up } & 67\end{array}$

$\begin{array}{ll}\text { Hotprinting } & 69\end{array}$

$\begin{array}{ll}\text { Double spacing } & 69\end{array}$

$\begin{array}{ll}\text { Justification } & 71\end{array}$

$\begin{array}{ll}\text { Saving and printing from the menu } & 72\end{array}$

Lesson Six: Using Special Effects $\quad \mathbf{7 4}$

Bold-printing text as you type it $\quad 79$

$\begin{array}{ll}\text { Bold-printing existing text } & 80\end{array}$

Deleting bold print $\quad 80$

Draft-printing text as you type it $\quad 81$

Draft-printing existing text $\quad 82$

Deleting draft print $\quad 82$

Enhanced-printing text as you type it $\quad 83$

Enhanced-printing existing text $\quad 83$

Deleting enhanced print $\quad 84$

Alphanumeric-underlining text as you type it $\quad 85$

$\begin{array}{ll}\text { Alphanumeric-underlining existing text } & 85\end{array}$

Deleting alphanumeric underline $\quad 86$

Underlining text as you type it $\quad 86$

$\begin{array}{ll}\text { Underlining existing text } & 87\end{array}$

Deleting underline $\quad 87$

$\begin{array}{ll}\text { Underlining and bold-printing text } & 87\end{array}$

$\begin{array}{ll}\text { Strikeout text as you type it } & 88\end{array}$

$\begin{array}{ll}\text { Strikeout existing text } & 89\end{array}$

$\begin{array}{ll}\text { Superscripting text } & 90\end{array}$

Subscripting text $\quad 91$

$\begin{array}{ll}\text { Deleting subscript or superscript } & 92\end{array}$

$\begin{array}{ll}\text { Changing the pitch } & 93\end{array}$

Changing the font $\quad 94$

$\begin{array}{ll}\text { Lesson Seven: Moving Text } & 97\end{array}$

$\begin{array}{lr}\text { Moving text } & 99\end{array}$

$\begin{array}{lr}\text { Highlighting text } & 100\end{array}$

$\begin{array}{ll}\text { De-highlighting text } & 101\end{array}$

$\begin{array}{ll}\text { Practising moving text } & 101\end{array}$

$\begin{array}{ll}\text { Cancelling a move } & 102\end{array}$

$\begin{array}{ll}\text { Saving and printing } & 102\end{array}$ 
Lesson Eight: Copying, Deleting and Restoring Text 107

$\begin{array}{ll}\text { Copying text } & 108\end{array}$

Deleting text $\quad 112$

$\begin{array}{ll}\text { Cancelling a copy or delete operation } & 112\end{array}$

$\begin{array}{ll}\text { Restoring text } & 112\end{array}$

$\begin{array}{ll}\text { Saving } & 113\end{array}$

$\begin{array}{ll}\text { Lesson Nine: Searching for and Replacing Text } & 114\end{array}$

$\begin{array}{ll}\text { Searching for text } & 115\end{array}$

$\begin{array}{ll}\text { Searching for text again } & 116\end{array}$

$\begin{array}{ll}\text { Signifying the case } & 117\end{array}$

$\begin{array}{ll}\text { Replacing text } & 118\end{array}$

$\begin{array}{ll}\text { Discretionary replacing text } & 118\end{array}$

$\begin{array}{ll}\text { Globally replacing text } & 119\end{array}$

$\begin{array}{ll}\text { Saving and printing } & 120\end{array}$

Lesson Ten: Page Length, Page Breaks, Go To Page, Page Combine, Printing

Selected Pages, Printing Copies

Setting the page length from the Modify Document

$\begin{array}{ll}\text { Defaults screen } & 124\end{array}$

$\begin{array}{ll}\text { Setting the page length from within the document } & 124\end{array}$

$\begin{array}{ll}\text { Making a page break } & 126\end{array}$

$\begin{array}{ll}\text { Go to page } & 127\end{array}$

$\begin{array}{ll}\text { Printing your five page document } & 129\end{array}$

$\begin{array}{ll}\text { Combining two pages } & 130\end{array}$

$\begin{array}{ll}\text { Preparation for printing practice } & 131\end{array}$

$\begin{array}{ll}\text { Saving } & 132\end{array}$

Printing a single page 132

$\begin{array}{ll}\text { Printing two copies } & 133\end{array}$

$\begin{array}{ll}\text { Printing pages } 2 \text { and } 3 & 133\end{array}$

Lesson Eleven: Spelling Checker and Thesaurus 135

$\begin{array}{ll}\text { Create document } & 135\end{array}$

Spell-check a section of a document 136

Delete entry from custom dictionary 139

$\begin{array}{ll}\text { Spell-check from Main Menu } & 139\end{array}$

Using the Thesaurus $\quad 141$

Lesson Twelve: Housekeeping $\quad 144$

$\begin{array}{ll}\text { What is housekeeping? } & 144\end{array}$

Making backups $\quad 144$

The file directory $\quad 146$

$\begin{array}{ll}\text { Copying a document } & 146\end{array}$

Moving a document $\quad 149$

Deleting a document 150

Renaming a document $\quad 152$

Printing the document summary screens 153

Searching for document summary screens $\quad 155$

Restoring a backed up document 156

$\begin{array}{ll}\text { MultiMate Advantage II Quick Reference Guide } & 159\end{array}$

$\begin{array}{ll}\text { Index } & 162\end{array}$ 
Joanna Gosling graduated in 1982 with an Honours degree in French from Hull University. After a series of posts in London, during which time she wrote her first book French Tarts published by Octopus, she embarked on a full-time career as a word processing trainer and writer. In the last two years she has written a number of books on word processing including Mastering Word Processing, 2nd edition for Macmillan Education in conjunction with her father, Peter Gosling, who has been a Macmillan author for over ten years. Now married to a geo-physicist, Joanna lives in Dubai where she is continuing her writing career which includes, apart from the current Macmillan Modern Office series, regular articles for locally produced magazines. 


\section{ACKNOWLEDGEMENTS}

Ashton-Tate and the Ashton-Tate logo are registered trademarks of Ashton-Tate Corporation.

MultiMate Advantage II is a trademark of Ashton-Tate Corporation. 